\title{
Qualidade de vida e fatores associados em trabalhadores do setor bancário
}

\author{
Quality of life and associated factors of banking industry professionals
}

\author{
Edilaine Alves Nunes', Claudio Henrique Meira Mascarenhas²
}

\begin{abstract}
RESUMO | Contexto: Com a intensificação do ritmo de trabalho dos bancários aumenta o risco do surgimento de distúrbios osteomusculares, dentre outros fatores que podem comprometer a qualidade de vida do trabalhador. Objetivo: Verificar a associação dos fatores sociodemográficos, ocupacionais, de estilo de vida e sintomas osteomusculares com a qualidade de vida dos bancários do município de Jequié (Bahia). Métodos: Trata-se de um estudo transversal, realizado com 111 voluntários, os quais responderam um questionário autoaplicado, abordando informações sociodemográficas, ocupacionais, de estilo de vida, de sintomas osteomusculares e de qualidade de vida. Resultados: Observou-se diferença estatisticamente significativa entre os indivíduos com maior comprometimento no domínio físico e os sintomas nos 12 meses, nos últimos 7 dias, afastamento das atividades, procura por assistência e estilo de vida. Constatou-se associação estatisticamente significativa entre o domínio psicológico e o estilo de vida, assim como entre o domínio relações sociais e as variáveis sexo, sintomas nos últimos 7 dias e afastamento das atividades. Conclusão: $\mathrm{O}$ maior comprometimento nos domínios físico e relações sociais da qualidade de vida estiveram associados à presença de sintomas musculoesqueléticos entre os bancários. O estilo de vida classificado como ruim apresentou associação com o maior comprometimento nos domínios físico e psicológico nesse grupo de trabalhadores. Espera-se que este estudo possa servir de base para o desenvolvimento de estratégias voltadas não só para promoção de saúde, mas também para a realização de intervenções sobre as variáveis que influenciam na qualidade de vida desse grupo de trabalhadores.
\end{abstract}

Palavras-chave I qualidade de vida; saúde do trabalhador; saúde pública.

ABSTRACT | Context: The higher the work rhythm of bankers, the higher the risk of osteomuscular disorders and other factors that may compromise the employee's quality of life. Objective: To verify the association of sociodemographic, occupational, lifestyle factors, and osteomuscular symptoms with quality of life of bankers from the city of Jequié (Bahia, Brazil). Methods: A cross-sectional study was carried out with 111 volunteers that answered a self-administered questionnaire regarding sociodemographic, occupational, lifestyle, osteomuscular symptoms, and quality of life information. Results: A statistically significant difference was found between subjects presenting greater impairment on the physical domain and symptoms in 12 months, during the last 7 days, leave of absence, care seeking, and life style. A statistically significant association of the psychological domain with life style was observed, as well as between the social relationship domain with the variables sex, symptoms during the last 7 days, and leave of absence. Conclusion: The greater impairment in the physical and social relationship of quality of life domains were associated with the occurrence of musculoskeletal symptoms among bankers. Poor lifestyle was associated with higher compromise in the physical and psychological domains in this group of employees. We hope this study can be used as a basis to develop strategies focused not only on health promotion, but also on the conduction of interventions focused on variables that impact the quality of life of this group of professionals.

Keywords I quality of life; occupational health; public health. 


\section{INTRODUÇÃO}

O avanço tecnológico impôs mudanças no processo de trabalho dos bancários, sendo que antes da informatização os caixas trabalhavam com máquinas que armazenavam os dados digitados que precisavam ser transmitidos após o fechamento das agências. A partir da informatização desse setor, cada caixa passou a operar com um terminal de computador, o que agilizou o tempo de atualização dos dados, reduzindo o tempo de atendimento e intensificando o ritmo de trabalho ${ }^{1,2}$, resultando em um aumento de funções manuais de conteúdo e repetitivas ${ }^{3}$.

O trabalho bancário exige habilidade e precisão em suas atividades. Por isso, requer um profissional com uma concepção mais generalista, detentor de habilidades para lidar com situações imprevistas, subjetivas e diversificadas, apto para manejar máquinas com rapidez e destreza nos movimentos corporais, e que cumpra as metas de produtividade estabelecidas pela gerência ${ }^{4,5}$.

Perante as demandas do trabalho surge o risco de aparecimento dos distúrbios osteomusculares relacionado ao trabalho, definidos como danos causados pela sobrecarga de atividades ao sistema musculoesquelético, surgindo de maneira insidiosa e caracterizada pelo aparecimento de sintomas concomitantes ou não - tais como dor, parestesia, sensação de peso e fadiga —, de aparecimento insidioso, geralmente nos membros superiores, o que pode comprometer a qualidade de vida do indivíduo ${ }^{6,7}$.

A qualidade de vida é vista como uma construção subjetiva, multidimensional, composta por elementos positivos e negativos, ampliando o espectro de análise dos processos envolvidos na perspectiva da ecologia humana e da investigação das conexões entre as múltiplas dimensões da relação entre saúde e trabalho ${ }^{8}$. Abordar a qualidade de vida no ambiente de trabalho pode trazer melhorias para a saúde dos profissionais, sendo uma necessidade de vital importância para que o trabalhador tenha condições para desempenhar com eficácia suas tarefas, de modo que todos saiam ganhando: empregador e colaborador?.

Nessa perspectiva, este estudo teve como objetivo verificar a associação dos fatores sociodemográficos, ocupacionais, de estilo de vida e sintomas osteomusculares com a qualidade de vida dos bancários do município de Jequié (Bahia).

\section{MÉTODOS}

Trata-se de um estudo descritivo-analítico com delineamento transversal, realizado com bancários do município de Jequié (Bahia). Foram incluídos no estudo bancários lotados no município de Jequié, com mais de 12 meses de trabalho no setor, sendo excluídos aqueles que estavam afastados de suas atividades durante o período da coleta de dados. Dessa forma, de um total de 165 bancários, 16 encontravam-se afastados das atividades e 38 recusaram participar do estudo, perfazendo um total de 111 voluntários para a pesquisa.

Os dados foram coletados entre os meses de julho e agosto de 2015. As informações foram registradas pelo voluntário da pesquisa em um questionário composto por cinco blocos de informações. Os questionários foram entregues aos participantes do estudo e recolhidos em datas pré-agendadas. Os indivíduos que não devolveram os questionários em até três retornos foram considerados como recusa à pesquisa.

O primeiro bloco do questionário consistiu em questões sociodemográficas, tais como sexo, idade, cor, situação conjugal, escolaridade e renda. $\mathrm{O}$ segundo bloco tratou de questões relacionadas à ocupação, como tempo de serviço, carga horária semanal e realização de outra atividade remunerada.

O terceiro bloco avaliou o estilo de vida por meio do questionário "estilo de vida fantástico". Esse é um instrumento genérico que foi desenvolvido no Departamento de Medicina Familiar da Universidade McMaster, no Canadá, por Wilson e Ciliska, em 1984, sendo traduzido para o português e validado no Brasil por Anez, Reis e Petroski ${ }^{10}$, no ano de 2008. É um instrumento autoadministrado que considera o comportamento dos indivíduos no último mês e cujos resultados permitem determinar a associação entre o estilo de vida e a saúde. $\mathrm{O}$ instrumento possui 25 questões divididas em 9 domínios, que são:

1. família e amigos;

2. atividade física;

3. nutrição;

4. cigarro e drogas;

5. álcool;

6. sono, cinto de segurança, estresse e sexo seguro;

7. tipo de comportamento;

8. introspecção; e

9. trabalho. 
A partir da análise desses nove domínios, o estilo de vida pode ser classificado em regular, bom, muito bom e excelente.

O quarto bloco consistiu de informações referentes aos sintomas osteomusculares, no qual foi utilizado o Nordic Musculoskeletal Questionnaire (NMQ), desenvolvido com a proposta de padronizar a mensuração de relato de sintomas osteomusculares e, assim, facilitar a comparação dos resultados entre os estudos ${ }^{11}$. Nesse questionário o respondente relatou a ocorrência dos sintomas considerando os últimos 12 meses e os últimos 7 dias precedentes à entrevista, bem como relatou a ocorrência de afastamento das atividades rotineiras no último ano em decorrência de sintomas musculoesqueléticos em uma ou mais das dez regiões anatômicas abordadas no questionário - pescoço, ombro, cotovelo, antebraço, punho/mão/dedos, dorsal, lombar, quadril/coxa, joelho e tornozelo/pé.

O quinto bloco avaliou a qualidade de vida a partir da aplicação do questionário WHOQOL-Bref. Esse questionário foi desenvolvido pela Organização Mundial da Saúde (OMS), e é constituído de 26 perguntas — sendo as duas primeiras sobre a qualidade de vida geral. As respostas seguem uma escala de Likert de 1 a 5 - quanto maior a pontuação melhor a qualidade de vida. Além das duas primeiras questões - 1 e 2 -, o instrumento tem 24 questões que compõem 4 domínios - físico, psicológico, relações sociais e meio ambiente ${ }^{8}$.

O domínio físico consiste de seis questões: dor e desconforto; energia e fadiga; sono e repouso; mobilidade; atividades da vida cotidiana; dependência de medicação ou de tratamentos; e capacidade de trabalho. O domínio psicológico é composto por seis questões, propostas para avaliar: os sentimentos positivos; o pensar, o aprender, a memória e a concentração; a autoestima; a imagem corporal e a aparência; os sentimentos negativos; e a espiritualidade, a religião e as crenças pessoais ${ }^{8}$.

No aspecto das relações sociais, analisa-se: as relações pessoais; o apoio social; e a atividade sexual. No domínio meio ambiente, o questionário é composto por oito questões, que analisam: a segurança física e a proteção; o ambiente do lar; os recursos financeiros; os cuidados de saúde; a oportunidade de adquirir novas informações e habilidades; a participação em e a oportunidade de lazer; o ambiente físico; e o transporte ${ }^{8}$.
A análise dos dados foi realizada por meio do programa Statistical Package for the Social Sciences (SPSS), versão 21.0, sendo que, inicialmente, para as variáveis sociodemográficas, ocupacionais, estilo de vida, sintomas osteomusculares e qualidade de vida, foram utilizadas a estatística descritiva com determinação das médias e desvio padrão e as variáveis quantitativas; para as variáveis categóricas, foram utilizadas as frequências absolutas e relativas.

Para verificar a existência de associação das variáveis sociodemográficas, ocupacionais, estilo de vida e sintomas osteomusculares com a qualidade de vida foram utilizados o teste $\chi^{2}$ e o teste Exato de Fischer - para valores esperados menores que 5 -, considerando nível de significância $\mathrm{p}<0,05$ e intervalo de confiança de $95 \%$ (IC95\%).

Esta pesquisa obedeceu às normas éticas da Resolução 466/2012 do Conselho Nacional de Saúde e todos os participantes assinaram um termo de consentimento livre e esclarecido. O estudo foi aprovado pelo Comitê de Ética em Pesquisa da Universidade Estadual do Sudoeste da Bahia (CEP/UESB), sob o parecer 1.163.870.

\section{RESULTADOS}

A partir da análise dos dados, observou-se que a maioria dos bancários era do sexo masculino (64,9\%) e com idade inferior ou igual a 35 anos $(54,1 \%)$ - sendo que a média de idade foi de $38,32( \pm 11,18)$ anos, variando entre $18 \mathrm{e}$ 61 anos. A cor parda foi relatada por $52,3 \%$ dos participantes e 70,3\% possuíam união estável.

No que se refere à escolaridade, a maioria dos bancários referiram possuir ensino superior completo $(53,2 \%)$, renda menor ou igual a 5 salários-mínimos $(58,6 \%)$ e tempo de serviço menor ou igual a 8 anos $(52,3 \%)$. A média de tempo de serviço foi de $13,8( \pm 11,7)$ anos, variando entre 1 e 41 anos. A maioria possuía carga horária semanal maior que 30 horas (58,6\%), e 91,9\% afirmaram não possuir outra atividade remunerada.

$\mathrm{Na}$ avaliação do estilo de vida, constatou-se que a maioria dos indivíduos $(62,2 \%)$ apresentava um estilo de vida classificado como "muito bom". Os dados supracitados estão descritos na Tabela 1.

$\mathrm{Na}$ avaliação dos sintomas de distúrbios musculoesqueléticos, observou-se que, independente da 
Tabela 1. Distribuição das variáveis sociodemográficas, ocupacionais e estilo de vida dos bancários investigados. Jequié (BA), Brasil, 2016.

\begin{tabular}{|c|c|c|}
\hline Variáveis & $\mathrm{n}$ & $\%$ \\
\hline \multicolumn{3}{|l|}{ Sexo } \\
\hline Feminino & 39 & 35,1 \\
\hline Masculino & 72 & 64,9 \\
\hline \multicolumn{3}{|l|}{ Idade (anos) } \\
\hline$\leq 35$ & 60 & 54,1 \\
\hline$>35$ & 51 & 45,9 \\
\hline \multicolumn{3}{|l|}{ Cor } \\
\hline Branca & 46 & 41,4 \\
\hline Parda & 58 & 52,3 \\
\hline Negra & 7 & 6,3 \\
\hline \multicolumn{3}{|l|}{ Situação conjugal } \\
\hline Sem união estável & 33 & 29,7 \\
\hline Com união estável & 78 & 70,3 \\
\hline \multicolumn{3}{|l|}{ Escolaridade } \\
\hline Ensino médio completo & 16 & 14,4 \\
\hline Ensino superior completo & 59 & 53,2 \\
\hline Pós graduado & 36 & 32,4 \\
\hline \multicolumn{3}{|l|}{ Renda (salários-mínimos) } \\
\hline$\leq 5$ & 65 & 58,6 \\
\hline$>5$ & 46 & 41,4 \\
\hline \multicolumn{3}{|l|}{ Tempo de serviço (anos) } \\
\hline$\leq 8$ & 58 & 52,3 \\
\hline$>8$ & 53 & 47,7 \\
\hline \multicolumn{3}{|l|}{ Carga horária semanal (horas) } \\
\hline$\leq 30$ & 46 & 41,4 \\
\hline$>30$ & 65 & 58,6 \\
\hline \multicolumn{3}{|c|}{ Exerce outra atividade remunerada } \\
\hline Não & 102 & 91,9 \\
\hline Sim & 9 & 8,1 \\
\hline \multicolumn{3}{|l|}{ Estilo de vida } \\
\hline Excelente & 7 & 6,3 \\
\hline Muito bom & 69 & 62,2 \\
\hline Bom & 35 & 31,5 \\
\hline
\end{tabular}

região afetada, 78,4\% dos participantes apresentaram sintomas musculoesqueléticos nos últimos 12 meses e $63,1 \%$ referiram esses sintomas nos últimos 7 dias. No que diz respeito à assistência profissional e aos afastamentos, $41,4 \%$ relataram terem procurado assistência à saúde em função dos sintomas e $28,8 \%$ se afastaram das suas atividades profissionais em consequência desses sintomas (Figura 1).

Os resultados referentes aos sintomas musculoesqueléticos nos últimos 12 meses, nos últimos 7 dias e ao afastamento das atividades pelo acometimento das diferentes regiões corporais estão expostos na Figura 2. Verificou-se que as regiões mais acometidas nos últimos 12 meses foram: pescoço $(49,5 \%)$, lombar e ombro $(45,6 \%)$ e punho/mão/dedos $(35,1 \%)$. Em relação aos sintomas nos últimos 7 dias, observou-se que as regiões mais acometidas foram: lombar $(34,2 \%)$, pescoço e ombro (32,4\%) e punho/mão/dedos $(25,2 \%)$. As principais regiões responsáveis pelos afastamentos foram: ombro $(16,2 \%)$, lombar $(12,6 \%)$, e punho/mão/ dedos $(11,7 \%)$.

Em relação à qualidade de vida, observou-se que o domínio psicológico obteve o escore médio mais alto $(78,19)$, seguido pelo domínio das relações sociais $(76,87)$, domínio físico $(76,44)$ e, por último, o domínio meio ambiente $(67,99)$. A descrição detalhada desses dados está exposta na Tabela 2.

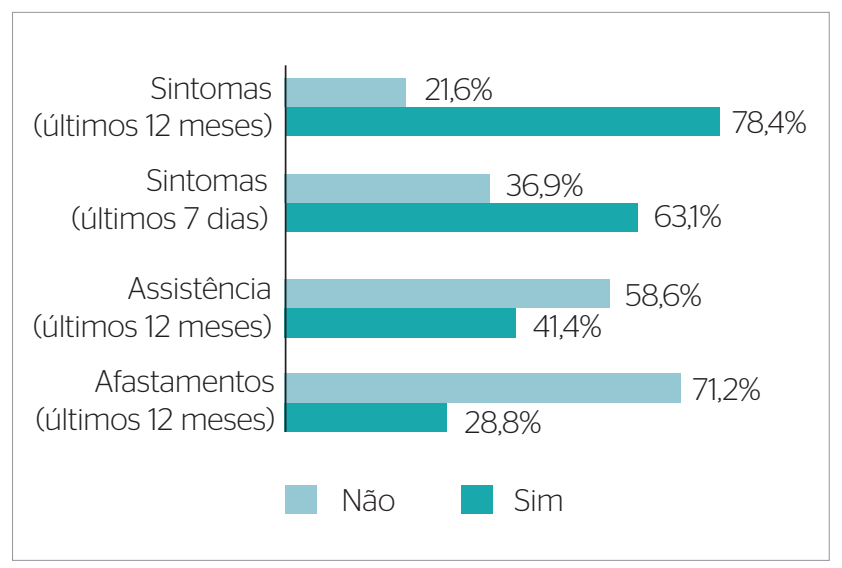

Figura 1. Distribuição dos sintomas musculoesqueléticos, afastamento das atividades e procura por profissional da área de saúde entre os bancários investigados. Jequié (BA), Brasil, 2016. 


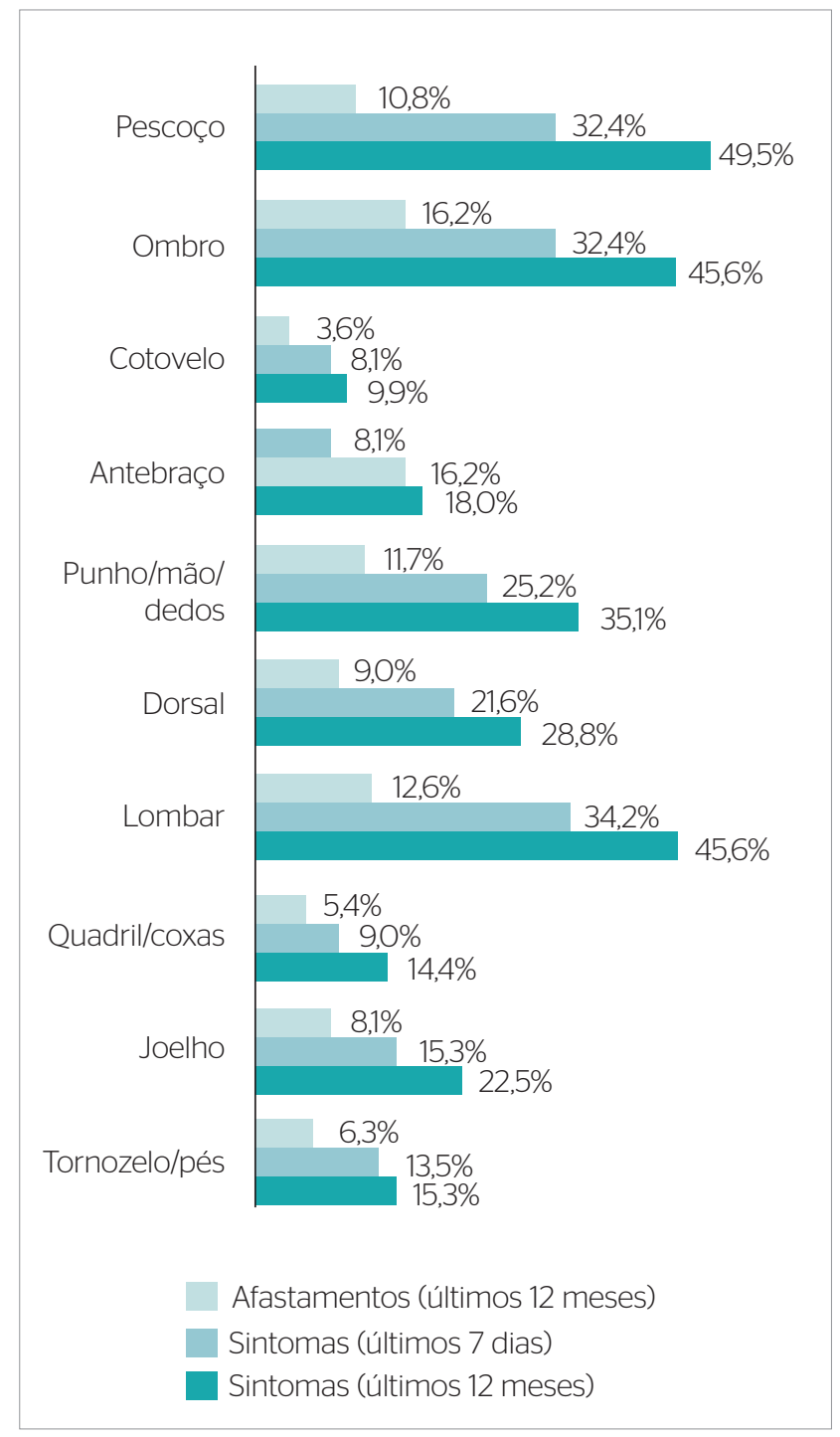

Figura 2. Distribuição dos sintomas musculoesqueléticos nos últimos 12 meses, últimos 7 dias e afastamento das atividades, nas diferentes regiões corporais, entre os bancários investigados. Jequié (BA), Brasil, 2016.
A partir da verificação da associação das variáveis encontrou-se diferença estatisticamente significativa entre o domínio físico e estilo de vida ( $\mathrm{p}=0,000)$, sintomas nos últimos 12 meses $(\mathrm{p}=0,004)$, sintomas nos últimos 7 dias $(\mathrm{p}=0,000)$, afastamento das atividades $(\mathrm{p}=0,002)$ e procura por assistência profissional $(\mathrm{p}=0,009)$. Dessa forma, notou-se que os indivíduos com maior comprometimento no domínio físico - ou seja, pontuação menor ou igual a 75 - apresentaram maior prevalência das variáveis estilo de vida "bom" (80,0\%), sintomas nos últimos 12 meses $(62,1 \%)$, nos últimos 7 dias $(68,6 \%)$, afastamento das atividades $(78,1 \%)$ e procura por assistência médica (69,6\%) (Tabela 3).

A verificação da associação do domínio psicológico com as demais variáveis do estudo mostrou diferença estatisticamente significativa entre esse domínio e o estilo de vida $(p=0,004)$. Dessa forma, verificou-se que os indivíduos com maior comprometimento no domínio psicológico pontuação menor ou igual a 79,17 — apresentaram maior prevalência do estilo de vida "bom" (82,9\%).

Na verificação da associação do domínio relações sociais e as demais variáveis observou-se diferença estatisticamente significativa entre esse domínio e o sexo $(p=0,026)$, os sintomas nos últimos 7 dias $(\mathrm{p}=0,001)$ e o afastamento das atividades $(p=0,003)$. Dessa forma, constatou-se que os indivíduos com maior comprometimento no domínio relações sociais - pontuação menor ou igual a 75 - apresentaram maior prevalência nas variáveis sexo feminino $(76,9 \%)$, sintomas nos últimos 7 dias $(74,3 \%)$ e afastamento das atividades $(84,4 \%)$ (Tabela 3$)$.

Não foram encontradas diferenças estatisticamente significativas entre o domínio meio ambiente e as demais variáveis estudadas.

Tabela 2. Distribuição dos resultados dos domínios do WHOQOL-Bref dos bancários investigados. Jequié (BA), Brasil, 2016.

\begin{tabular}{|c|c|c|c|c|c|}
\hline Domínios & Média & Mediana & DP & IC95\% & Min-Max \\
\hline Físico & 76,44 & 75,00 & 3,38 & 73,93-78,96 & $46,43-100$ \\
\hline Psicológico & 78,19 & 79,17 & 10,41 & 76,23-80,15 & 54,17-100 \\
\hline Relações sociais & 76,87 & 75,00 & 14,63 & $74,10-79,63$ & $16,67-100$ \\
\hline Meio ambiente & 67,99 & 65,63 & 12,69 & $65,60-70,38$ & $40,63-100$ \\
\hline
\end{tabular}

DP: desvio-padrão; IC95\%: índice de confiança de 95\%; Min-Max: mínimo-máximo. 
Tabela 3. Associação das variáveis sociodemográficas, ocupacionais, estilo de vida e sintomas de distúrbios musculoesqueléticos com o comprometimento da qualidade de vida dos bancários investigados. Jequié (BA), Brasil, 2016.

\begin{tabular}{|c|c|c|c|c|c|c|c|c|c|c|c|c|}
\hline \multirow{2}{*}{ Variável } & \multicolumn{3}{|c|}{ Físico $(\leq 75)$} & \multicolumn{3}{|c|}{ Psicológico ( $\leq 79,17)$} & \multicolumn{3}{|c|}{ Relações Sociais ( $\leq 75$ ) } & \multicolumn{3}{|c|}{ Meio Ambiente $(\leq 65,63)$} \\
\hline & $\mathrm{n}$ & $\%$ & Valor $\mathrm{p}$ & $\mathrm{n}$ & $\%$ & Valor $p$ & $\mathrm{n}$ & $\%$ & Valor $\mathrm{p}$ & $\mathrm{n}$ & $\%$ & Valor $\mathrm{p}$ \\
\hline \multicolumn{13}{|l|}{ Sexo } \\
\hline Feminino & 26 & 66,7 & \multirow{2}{*}{0,068} & 24 & 61,5 & \multirow{2}{*}{0,807} & 30 & 76,9 & \multirow{2}{*}{$0,026^{\star}$} & 24 & 61,5 & \multirow{2}{*}{0,063} \\
\hline Masculino & 35 & 48,6 & & 46 & 63,9 & & 40 & 55,6 & & 31 & 43,1 & \\
\hline \multicolumn{13}{|l|}{ Idade (anos) } \\
\hline$\leq 35$ & 32 & 53,3 & \multirow{2}{*}{0,710} & 35 & 58,3 & \multirow{2}{*}{0,263} & 35 & 58,3 & \multirow{2}{*}{0,263} & 29 & 48,3 & \multirow{2}{*}{0,781} \\
\hline$>35$ & 29 & 56,9 & & 35 & 68,6 & & 35 & 68,6 & & 26 & 51,0 & \\
\hline \multicolumn{13}{|l|}{ Cor } \\
\hline Branca & 26 & 56,5 & \multirow{3}{*}{0,192} & 30 & 65,2 & \multirow{3}{*}{0,895} & 30 & 65,2 & \multirow{3}{*}{0,895} & 25 & 54,3 & \multirow{3}{*}{0,429} \\
\hline Parda & 29 & 50,0 & & 36 & 62,1 & & 36 & 62,1 & & 28 & 48,3 & \\
\hline Negra & 6 & 85,7 & & 4 & 57,1 & & 4 & 57,1 & & 2 & 28,6 & \\
\hline \multicolumn{13}{|l|}{ Situação conjugal } \\
\hline Sem união & 18 & 54,5 & \multirow{2}{*}{0,536} & 18 & 54,5 & \multirow{2}{*}{0,183} & 22 & 66,7 & \multirow{2}{*}{0,385} & 13 & 39,4 & \multirow{2}{*}{0,247} \\
\hline Com união & 43 & 55,8 & & 52 & 67,5 & & 48 & 62,3 & & 41 & 53,2 & \\
\hline Escolaridade & & & & & & & & & & & & \\
\hline Médio & 8 & 50,0 & & 10 & 62,5 & & 9 & 56,3 & & 7 & 43,8 & \\
\hline Superior & 37 & 62,7 & $0,2 \mathrm{O}$ & 40 & 67,8 & 0,487 & 39 & 66,1 & 0,737 & 37 & 62,7 & 0,091 \\
\hline Pós-graduação & 16 & 44,4 & & 20 & 55,6 & & 22 & 61,1 & & 11 & 30,6 & \\
\hline Renda (salários-mí & & & & & & & & & & & & \\
\hline$\leq 5$ & 40 & 61,5 & (1) & 41 & 63,1 & Q997 & 40 & 61,5 & (692 & 34 & 52,3 & (1900 \\
\hline$>5$ & 21 & 45,7 & , & 29 & 63,0 & ו & 30 & 65,2 & , & 21 & 45,7 & 0,490 \\
\hline Tempo de serviço & & & & & & & & & & & & \\
\hline$\leq 8$ & 29 & 50,0 & & 34 & 58,6 & 310 & 34 & 58,6 & 310 & 29 & 50,0 & (9021 \\
\hline$>8$ & 32 & 60,4 &,$\angle 7 / 2$ & 36 & 67,9 & טוכ, & 36 & 67,9 & טוכ, & 26 & 49,1 & $0,9<1$ \\
\hline Carga horária (hor & & & & & & & & & & & & \\
\hline$\leq 30$ & 29 & 63,0 & 0150 & 29 & 63,0 & م097 & 30 & 65,2 & (0697 & 25 & 54,3 & 305 \\
\hline$>30$ & 32 & 49,2 & 徒, & 41 & 63,1 & 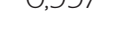 & 40 & 61,5 & 0,092 & 30 & 46,2 & נת, \\
\hline Outra atividade & & & & & & & & & & & & \\
\hline Não & 57 & 55,9 & (500 & 63 & 61,8 & 03 & 67 & 65,7 & ( & 53 & 52,0 & ค००7 \\
\hline Sim & 4 & 44,4 & , & 7 & 77,8 & U, & 3 & 33,3 & 0,034 & 2 & 22,2 & ו \\
\hline Estilo de vida & & & & & & & & & & & & \\
\hline Excelente & 1 & 14,3 & & 3 & 42,9 & & 3 & 42,9 & & 3 & 42,9 & \\
\hline Muito bom & 32 & 46,4 & $0,000^{*}$ & 38 & 55,1 & $0,004^{*}$ & 44 & 63,8 & 0,510 & 34 & 49,3 & 0,915 \\
\hline Bom & 28 & 80,0 & & 29 & 82,9 & & 23 & 65,7 & & 18 & 51,4 & \\
\hline
\end{tabular}


Tabela 3. Continuação.

\begin{tabular}{|c|c|c|c|c|c|c|c|c|c|c|c|c|}
\hline \multirow{2}{*}{ Variável } & \multicolumn{3}{|c|}{ Físico $(\leq 75)$} & \multicolumn{3}{|c|}{ Psicológico $(\leq 79,17)$} & \multicolumn{3}{|c|}{ Relações Sociais $(\leq 75)$} & \multicolumn{3}{|c|}{ Meio Ambiente $(\leq 65,63)$} \\
\hline & $n$ & $\%$ & Valor $p$ & $n$ & $\%$ & Valor $p$ & $n$ & $\%$ & Valor $\mathrm{p}$ & $n$ & $\%$ & Valor $\mathrm{p}$ \\
\hline \multicolumn{13}{|c|}{ Sintomas 12 meses } \\
\hline $\mathrm{Sim}$ & 54 & 62,1 & \multirow{2}{*}{$0,004^{*}$} & 56 & 64,4 & \multirow{2}{*}{0,588} & 57 & 65,5 & \multirow{2}{*}{0,308} & 47 & 54,0 & \multirow{2}{*}{0,073} \\
\hline Não & 7 & 29,2 & & 14 & 58,3 & & 13 & 54,2 & & 8 & 33,3 & \\
\hline \multicolumn{13}{|c|}{ Sintomas 7 dias } \\
\hline Sim & 48 & 68,6 & \multirow{2}{*}{$0,000^{*}$} & 47 & 67,1 & \multirow{2}{*}{0,245} & 52 & 74,3 & \multirow{2}{*}{$0,001^{\star}$} & 39 & 55,7 & \multirow{2}{*}{0,090} \\
\hline Não & 13 & 31,7 & & 23 & 56,1 & & 18 & 43,9 & & 16 & 39,0 & \\
\hline \multicolumn{13}{|c|}{ Afastamento } \\
\hline $\operatorname{Sim}$ & 25 & 78,1 & \multirow{2}{*}{$0,002^{*}$} & 23 & 71,9 & \multirow{2}{*}{0,221} & 27 & 84,4 & \multirow{2}{*}{$0,003^{*}$} & 20 & 62,5 & \multirow{2}{*}{0,082} \\
\hline Não & 36 & 45,6 & & 47 & 59,5 & & 43 & 54,4 & & 35 & 44,3 & \\
\hline \multicolumn{13}{|c|}{ Procura por assistência } \\
\hline $\operatorname{Sim}$ & 32 & 69,6 & \multirow{2}{*}{$0,009^{*}$} & 32 & 69,6 & \multirow{2}{*}{0,232} & 32 & 69,6 & \multirow{2}{*}{0,232} & 27 & 58,7 & \multirow{2}{*}{0,105} \\
\hline Não & 29 & 44,6 & & 38 & 58,5 & & 38 & 58,5 & & 28 & 43,1 & \\
\hline
\end{tabular}

*Valor p estatisticamente significante.

\section{DISCUSSÃO}

O presente estudo apontou que independente da região afetada, 78,4\% dos participantes da pesquisa apresentaram sintomas musculoesqueléticos nos últimos 12 meses e $63,1 \%$ referiram esses sintomas nos últimos 7 dias. Em um estudo realizado por Brandão, Horta e Tomasi ${ }^{12}$ na cidade de Pelotas, Rio de Janeiro, com 502 bancários, foram encontradas menores prevalências de sintomas musculoesqueléticos quando comparadas ao presente estudo, sendo $59,6 \%$ nos últimos 12 meses e 42,6\% nos últimos 7 dias. Segundo esses autores, a demanda e a organização do trabalho bancário estão diretamente inseridas em um contexto com uma grande possibilidade de desencadear um distúrbio osteomuscular.

Para Bomgiorno e Tokars ${ }^{13}$, alguns elementos podem ser apontados como sendo responsáveis pelo surgimento dos sintomas osteomusculares em bancários, tais como: mobiliários e equipamentos mal projetados, longas jornadas de trabalho, inexistências de intervalos para descanso, excesso de movimentos repetitivos, estresse, tensão, alta exigência e sobrecarga de trabalho.

No que diz respeito aos afastamentos, $28,8 \%$ dos entrevistados afirmaram ter se ausentado das atividades profissionais em consequência de sintomas osteomusculares. Nesse mesmo contexto, no estudo realizado por Brandão, Horta e Tomasi ${ }^{12}$, foi observado que $18,9 \%$ dos bancários acometidos por tais sintomas se afastaram do trabalho. A elevada percentagem encontrada no presente estudo mostra a gravidade desses distúrbios relacionados ao trabalho, o que pode interferir na saúde desses trabalhadores, e também repercutir nas atividades profissionais dos mesmos.

No presente estudo, ficou evidenciado que a maioria dos entrevistados que relatou sintomas osteomusculares não buscou assistência à saúde. Esse é um dado preocupante, pois a negligência de um problema aparentemente simples pode, a médio e longo prazo, gerar maiores prejuízos pessoais e profissionais a esses trabalhadores.

Quanto ao acometimento dos sintomas por regiões corporais, foi constatado que, nos últimos 12 meses, as regiões mais acometidas foram o pescoço, a lombar e o ombro. Essa constatação também pode ser observada no estudo de Bomgiorno e Tokars ${ }^{13}$, realizado em Curitiba, Paraná, com 57 bancários. $\mathrm{O}$ acometimento de tais regiões pode ser explicado pela realização de posturas forçadas e prolongadas, pelos movimentos repetitivos de membros superiores ou gestos realizados em contração estática e pela 
organização do trabalho dos bancários, constituindo, assim, fatores geradores de distúrbios osteomusculares, principalmente se associados ao uso de equipamentos inadequados ${ }^{14,15}$.

Em relação à avaliação da qualidade de vida dos bancários - realizada a partir do WHOQOL-Bref —, o escore varia de 0 a 100, no qual os maiores escores médios indicam melhor avaliação desse construto. A partir dos resultados, foram observados que os escores médios dos domínios variaram de 67,99 a 78,19 , dos quais o domínio psicológico obteve o maior escore médio, seguido pelos domínios relações sociais, físico e meio ambiente. No estudo realizado por Maier e Santos Junior ${ }^{16}$, em Ponta Grossa, Paraná, com 28 bancários, em 2009, também foram encontrados melhores escores nos domínios relações sociais $(69,12)$ e psicológico $(66,83)$, e os piores escores nos domínios meio ambiente $(60,35)$ e físico $(56,77)$.

Embora alguns estudos mostrem que a atividade bancária possa trazer ao trabalhador um comprometimento psicológico em função da insegurança, da impotência e das incertezas propostas pelas empresas - assim como exigências impostas pela produtividade, dentre outros fatores ${ }^{17}$ - observou-se no presente estudo uma elevada pontuação no domínio psicológico. Barros e Mendes ${ }^{18}$ afirmam que fatos como esse podem ser explicados a partir da psicodinâmica, ou seja, consiste numa estratégia defensiva do trabalhador que visa reduzir o sofrimento psíquico no trabalho por meio da utilização de mecanismos que protegem o sujeito contra o sofrimento causado pelas situações de trabalho geradoras de conflito, mantendo, assim, o equilíbrio psíquico e evitando o adoecimento.

O domínio das relações sociais foi o segundo mais bem pontuado. Para Barros e Mendes ${ }^{18}$, o bom índice nas relações sociais demonstra que existe um suporte social oferecido pelos colegas e gestores, o que possivelmente favorece a neutralização do sofrimento advindo da organização e das condições de trabalho, aliviando o estresse e a ansiedade do indivíduo.

O baixo índice encontrado no domínio físico, quando comparado aos domínios psicológico e relações sociais, pode estar relacionado com os tipos de atividades desenvolvidas por esses trabalhadores, uma vez que constituem, muitas vezes, em atividades repetitivas e que exige muito de uma biomecânica e ergonomia adequadas, além da alta prevalência de sintomas osteomusculares encontrados nesses trabalhadores.

Com relação ao domínio meio ambiente, este foi considerado o mais comprometido dentre os domínios de qualidade de vida. Dentre as questões que avaliam esse domínio, as mais comprometidas foram: recurso financeiro, ambiente e lar, e oportunidade de adquirir novas informações e habilidade.

Quanto à verificação da associação entre as variáveis independentes e os domínios do WHOQOL-Bref, contatou-se no domínio físico que as variáveis estilo de vida, sintomas nos últimos 12 meses, sintomas nos últimos 7 dias, afastamento das atividades e procura por assistência profissional apresentaram diferença estatisticamente significativa. A elevada prevalência dos sintomas musculoesqueléticos influencia diretamente nas facetas do domínio físico, uma vez que estas avaliam dor, desconforto e capacidade de trabalho, estando diretamente relacionado com a qualidade de vida do indivíduo. Já a presença de afastamento do trabalho e procura por assistência à saúde em função dos sintomas estão estreitamente relacionadas às facetas de domínio que se referem às atividades cotidianas e à dependência de medicação.

Verificou-se também a associação estatisticamente significativa entre o domínio psicológico e o estilo de vida, na qual se observou que quanto menor a classificação do estilo de vida, maior a proporção de indivíduos com comprometimento nos domínios físico e psicológico. $\mathrm{O}$ estilo de vida é compreendido como um conjunto de valores e atitudes adotados pelo sujeito em resposta ao seu ambiente social, cultural e econômico, bem como um padrão de comportamento que o indivíduo pode conferir ${ }^{19}$, possuindo necessidades de saúde distintas em cada fase da vida, que dependem da qualidade de interação entre as esferas biológica, psicológica e social, de acordo com a etapa de desenvolvimento ${ }^{20}$, havendo necessidade de orientações relacionadas ao estilo de vida, para que este influencie de forma positiva na saúde mental e física do sujeito ${ }^{21}$.

$\mathrm{Na}$ avaliação do domínio relações sociais, verificou-se associação estatisticamente significativa entre esse domínio e as variáveis sexo, sintomas nos últimos 7 dias e afastamento das atividades. O domínio das relações sociais avalia as relações pessoais, o apoio social e as relações sexuais. Boschco ${ }^{22}$ define relações sociais como sendo a maneira de agir no mundo, sendo renovada por meio de sua estilização. Logo, a presença do sintoma musculoesquelético implica na realização das atividades diárias ${ }^{23}$, alterando diversos aspectos da vida e contribuindo para o isolamento social ${ }^{24}$, o que interfere diretamente nas relações sociais. $\mathrm{O}$ afastamento do trabalho também evidenciado no estudo de Boschco altera diretamente as relações sociais do sujeito, 
implicando na saúde psicossocial do trabalhador e fragilizando o indivíduo ${ }^{22}$.

O presente estudo apresentou limitações relacionadas ao desenho epidemiológico empregado, uma vez que os estudos de corte transversal são restritos à identificação de associações, impossibilitando o estabelecimento da direção causal das relações encontradas, além de não permitir a realização de análises de temporalidade entre exposição e desfecho, não podendo apontar riscos. Outro fator a ser destacado foi o viés de seleção da amostra intencional, no qual 32\% da amostra inicial não participou do estudo por recusa ou afastamento das atividades no período pesquisado.

\section{CONCLUSÃO}

O presente estudo evidenciou uma elevada distribuição de sintomas de distúrbios musculoesqueléticos nos últimos 12 meses e nos últimos 7 dias, sendo que as regiões mais acometidas foram o pescoço, a lombar e o ombro. Em relação à qualidade de vida, observou-se que o domínio psicológico obteve o escore médio mais elevado, seguido pelos domínios relações sociais, físico e, por último, meio ambiente.
A partir da verificação da associação das variáveis com o comprometimento da qualidade de vida, foi possível observar diferença estatisticamente significativa entre os indivíduos com pontuação menor ou igual a 75 no domínio físico e os sintomas nos últimos 12 meses, nos últimos 7 dias, o afastamento das atividades, a procura por assistência e o estilo de vida. Verificou-se também a associação estatisticamente significativa entre o domínio psicológico e o estilo de vida. Quanto ao domínio relações sociais observou-se que os indivíduos com pontuação menor ou igual a 75 tiveram associação estatisticamente significativa com sexo, sintomas nos últimos 7 dias e afastamento das atividades. Contudo, pode-se afirmar que o estilo de vida e os sintomas osteomusculares possuem relação direta com a qualidade de vida.

Os resultados deste estudo é um passo essencial para que os profissionais atuantes na área possam tomar atitudes mais apropriadas, facilitando o manejo dos aspectos que comprometem a qualidade de vida, assim como possa servir de base para o desenvolvimento de estratégias voltadas não só para promoção da saúde, mas também para a realização de intervenções necessárias sobre as variáveis que influenciam a qualidade de vida desse grupo de trabalhadores.

\section{REFERÊNCIAS}

1. Segnini LRP. Mulheres no trabalho bancário: difusão tecnológica, qualificação e relações de gênero. São Paulo (SP): Edusp/ Fapesp, 1998.

2. Murofuse NT, Marziale MHP. Mudanças no trabalho e na vida de bancários portadores de lesões por esforços repetitivos: LER. Rev Latino-Am Enfermagem. 2001;9(4):19-25.

3. Rua MPA, Macedo RB, Seleme VB, Cerqueira MLW. Transtornos traumáticos cumulativos em bancários. Rev Bras Med Trab. 2010:8(2):60-7.

4. Martins LF. Estresse ocupacional e esgotamento profissional entre profissionais da atenção primária à saúde. 142 f. [Dissertação de Mestrado]. Curso de Psicologia, Pós-graduação, Universidade Federal de Juiz de Fora, Juiz de Fora, 2011.

5. Silva JL, Navarro VL. Organização do trabalho e saúde de trabalhadores bancários. Rev Latino-Am Enfermagem. [on-line]. 2012;20(2):[9 telas]. Disponível em: http://www.scielo.br/pdf/rlae/ v20n2/pt_03.pdf
6. Brasil. Instituto Nacional do Seguro Social. Ordem de Serviço INSS/ DSS n 606, de 5 de agosto de 1998. Aprova Norma Técnica sobre Distúrbios Osteomusculares Relacionados ao Trabalho - DORT. Diário Oficial da União, 1998. [acesso 2016 mar 15]. Disponível em: http://www.edmilsonbarros.adv.br/intra/mensagens/ORDEM\%20 DE\%2OSERVI\%C3\%870\%2OINSS\%20-\%2ODORT2.doc

7. Maeno M,Salerno V, Rossi DAG, Fuller R. Lesões por Esforços Repetitivos (LER), Distúrbios Osteomusculares Relacionados ao Trabalho (Dort). Departamento de ações programáticas estratégicas área técnica de saúde do trabalhador. Protocolos de atenção integral à Saúde do Trabalhador deComplexidade Diferenciada. Brasília/DF.Fevereiro 2006.

8. Fleck MPA. O instrumento de avaliação de qualidade de vida da Organização Mundial da Saúde (WHOQOL-100): características e perspectivas. Ciênc Saúde Coletiva. 2000;5(1):33-8.

9. Carvalho JF, Martins EPT, Lúcio L, Papandréa PJ. Qualidade de vida no trabalho e fatores motivacionais dos colaboradores nas organizações. Educação em Foco. 2013;7:21-31. 
10. Añez CRR, Reis RS, Petroski EL. Versão brasileira do questionário "Estilo de Vida Fantástico": tradução e validação para adultos jovens. Arq Bras Cardiol. 2008;91(2):102-9.

11. Pinheiro FA, Troccoli BT, Carvalho CV. Validação do Questionário Nórdico de Sintomas Osteomusculares como medida de morbidade. Rev Saúde Pública. 2002;36(3):307-12.

12. Brandão GB, Horta LH, Tomasi E. Sintomas de distúrbios osteomusculares em bancários de Pelotas e região: prevalência e fatores associados. Rev Bras Epidemiol. 2005;8(3):295-305.

13. Bomgiorno A, Tokars E. Prevalência dos Sintomas de Distúrbios Osteomusculares em bancários da cidade de Curitiba. Curitiba, 2011. [acesso 2016 mar 15]. Disponível em: http://tcconline.utp.br/ wp-content/uploads/2011/04/prevalencia-dos-sintomas-de-disturbiososteomusculares-em-bancarios-da-cidade-de-curitiba.pdf

14. Alexopoulos EC, Stathi I-C, Charizani F. Prevalence of musculoskeletal disorders in dentists. BMC Musculoskelet Disord. 2004;5:16.

15. Henríquez MG, Rivera CF, Eyzaguirre JM. Prevalência de transtornos músculoesqueléticos de coluna lumbar em trabajadoras y límites biomecânicos enel manejo de carga y pacientes. Cienc Trab. 2010;37:380-5.

16. Maier RC, Santos Junior G. Análise exploratória da qualidade de vida: um estudo com bancários na cidade de Ponta Grossa/PR. Ciências Sociais em Perspectiva. 2011;10(18):11-24.

17. Merlo ARC, Barbarini, N. Reestruturação produtiva no setor bancário brasileiro e sofrimento dos caixas executivos: um estudo de caso. Psicol Soc. 2002;14(1):103-22.
18. Barros PCR, Mendes AMB. Sofrimento psíquico no trabalho e estratégias defensivas dos operários terceirizados da construção civil. Psico-USF. 2003;8(1):63-70.

19. Perraccini MR, Fló CM. Fisioterapia teoria e prática: funcionalidade e envelhecimento. Rio de Janeiro: Guanabara Koogan, 2009.

20. Costa MCO, Bigras M. Mecanismos pessoais e coletivos de proteção e promoção da qualidade de vida para a infância e adolescência. Ciênc Saúde Coletiva. 2007;12(5):1101-9.

21. Maciel ES. Estilo de vida: a importância do estímulo por parte dos adultos. In: Boccaletto EM, Mendes RT, Vilarta R. (Org.). Estratégias de promoção da saúde do escolar: atividade física e alimentação saudável. Campinas: IPÊS, 2010. p. 93-9.

22. Boschco CR. Implicações do afastamento do trabalho por adoecimento na subjetividade do auxiliar de enfermagem. Curitiba. Dissertação [Mestrado em Psicologia] - Programa de Pós-graduação em Psicologia, Setor de Ciências Humanas, Letras e Artes da Universidade Federal do Paraná; 2011.

23. Meira-Mascarenhas $\mathrm{CH}$, Ornellas-Prado F, Henrique-Fernandes M. Dor musculoesquelética e qualidade de vida em agentes comunitários de saúde. Rev Salud Pública. 2012;14(4):668-80.

24. O'sullivan SB, Schimitz, TJ. Fisioterapia: avaliação e tratamento. 4. ed. São Paulo: Manole, 2004.

Endereço para correspondência: Edilaine Alves Nunes - Avenida Almenara, 477 - Planalto - CEP: 39915-000 - Mata Verde (MG), Brasil -

E-mail: edilainunes@gmail.com 